\title{
Multimodal Biometric Identification System Based On Iris \& Fingerprint
}

\author{
Ms. Priyanka S. Patil ${ }^{1}$, Prof.(Dr.) A. S. Abhyankar ${ }^{2}$ \\ ${ }^{1}$ (Department of E\&TC(Signal Processing), VIIT College of Engineering Pune, India ) \\ ${ }^{2}$ (Department of Computer Engineering, VIIT college of Engineering Pune, India)
}

\begin{abstract}
This paper is related to the development of an innovative multimodal biometric identification system. Unimodal biometric systems often face significant limitations due to sensitivity to noise intraclass variability and other factors. Multimodal biometric identification systems aim to fuse two or more physical or behavioral traits to provide optimal False Acceptance Rate (FAR) and False Rejection Rate (FRR), thus improving system accuracy and dependability. In greater detail, a Multimodal Biometric Identification System Based On Iris \& Fingerprint. Both biometric traits (Iris \& Fingerprint) are processed individually through all steps like segmentation, feature extraction \& matching. The multimodal system is fused using match level fusion at the verification stage on obtained matching score of iris and fingerprint. The performance of the biometric system shows improvement in the False Acceptance Rate (FAR) and False Reject Rate (FRR). The proposed multimodal system achieves interesting results with several commonly used databases.
\end{abstract}

Keywords - Unimodal biometrics, multimodal biometric systems, iris and fingerprint biometry.

\section{INTRODUCTION}

Biometrics, which refers to identify an individual based on his or her physiological or behavioral characteristics, has the capability to reliably distinguish between an authorized person and an imposter. Since biometric characteristics are distinctive, cannot be forgotten or lost, and the person to be authenticated needs to be physically present at the point of identification. Most biometric systems deployed in real-world applications are unimodal, i.e., they rely on the evidence of a single source of information for authentication (e.g., single fingerprint or Iris). unimodal biometric systems often face significant limitations due to sensitivity to noise, intraclass variability, data quality, non universality, and other factors. Limitations of unimodal biometric systems can be overcome by using multimodal biometric systems. Multimodal biometrics refers to the use of a combination of two or more biometric modalities in a verification / identification system. The goal of multibiometrics is to reduce False accept rate (FAR), False reject rate (FRR).

A multi-biometric system can be classified into one of the 4 categories:

1.Multi-algorithm 2.Multi-instance

3.Multi-sample 4.Multimodal.

Multibiometrics data can be combined at different levels fusion:

1.Data-sensor level 2. Feature extraction level

3.Matching level 4. Decision level.

\section{RELATED WORK}

A variety of articles can be found, which propose different approaches for unimodal and multimodal biometric systems. Traditionally, unimodal biometric systems have many limitations. Multimodal biometric systems are based on different biometric features and/or introduce different fusion algorithms for these features. Many researchers have demonstrated that the fusion process is effective, because fused scores provide much better discrimination than individual scores. Such results have been achieved using a variety of fusion techniques. An unimodal fingerprint verification and classification system is presented in [16]. The system is based on a feedback path for the feature-extraction stage, followed by a feature-refinement stage to improve the matching performance. This improvement is illustrated in the contest of a minutiae-based fingerprint verification system. The Gabor filter is applied to the input image to improve its quality.

Ratha et al. [17] proposed a unimodal distortion-tolerant fingerprint authentication technique based on graph representation. Using the fingerprint minutiae features, a weighted graph of minutiae is constructed for both the query fingerprint and the reference fingerprint. The proposed algorithm has been tested with excellent results on a large private database with the use of an optical biometric sensor. Concerning iris recognition systems in [14], the Gabor filter and 2-D wavelet filter are used for feature extraction. This method is invariant to translation and rotation and is tolerant to illumination. The classification rate on using the Gabor is $98.3 \%$ and the accuracy with wavelet is $82.51 \%$ on the Institute of Automation of the Chinese Academy of Sciences (CASIA) database. In the approach proposed in [13], multichannel and Gabor filters have been used to capture 
local texture information of the iris, which are used to construct a fixed-length feature vector. The results obtained were FAR $=0.01 \%$ and FRR $=2.17 \%$ on CASIA database. Generally, unimodal biometric recognition systems present different drawbacks due its dependency on the unique biometric feature. For example, feature distinctiveness, feature acquisition, processing errors, and features that are temporally unavailable can all affect system accuracy. A multimodal biometric system should overcome the aforementioned limits by integrating two or more biometric features.

Conti et al. [20] proposed a multimodal biometric system using two different fingerprint acquisitions. The matching module integrates fuzzy-logic methods for matching-score fusion. Experimental trials using both decision-level fusion and matching-score-level fusion were performed. Experimental results have shown an improvement of $6.7 \%$ using the matching score- level fusion rather than a monomodal authentication system. Besbes et al. [12] proposed a multimodal biometric system using fingerprint and iris features. They use a hybrid approach based on: 1) fingerprint minutiae extraction and 2) iris template encoding through a mathematical representation of the extracted iris region. This approach is based on two recognition modalities and every part provides its own decision. The final decision is taken by considering the unimodal decision through an "AND" operator. No experimental results have been reported for recognition performance.

Aguilar et al. [21] proposed a multibiometric method using a combination of fast Fourier transform (FFT) and Gabor filters to enhance fingerprint imaging. Successively, a novel stage for recognition using local features and statistical parameters is used. The proposed system uses the fingerprints of both thumbs. Each fingerprint is separately processed; successively, the unimodal results are compared in order to give the final fused result. The tests have been performed on a fingerprint database composed of 50 subjects obtaining FAR $=$ $0.2 \%$ and $\mathrm{FRR}=1.4 \%$.

Subbarayudu and Prasad [22] presented experimental results of the unimodal iris system, unimodal palmprint system, and multibiometric system (iris and palmprint). The system fusion utilizes a matching scores feature in which each system provides a matching score indicating the similarity of the feature vector with the template vector. The experiment was conducted on the Hong Kong Polytechnic University Palmprint database. A total of 600 images are collected from 100 different subjects. In contrast to the approaches found in literature and detailed earlier, the proposed approach introduces an innovative idea to unify and homogenize the final biometric descriptor using two different strong features - the fingerprint and the iris. In opposition to [23], this paper shows the improvements introduced by adopting the fusion process at the template level as well as the related comparisons against the unimodal elements and the classical matching-score fusion-based multimodal system. In addition, the system proposed in this paper has been tested on the official fingerprint FVC2002 DB2 and iris BATH databases [18], [24].

\section{MULTIMODAL BIOMETRIC IDENTIFICATION SYSTEM}

In this paper, we introduce a frequency approach [17], to generate a unified homogeneous template for fingerprint and iris features. In greater detail, the proposed approach performs fingerprint matching using the segmented regions (Regions Of Interests, ROIs) surrounding fingerprint minutia points[11]. On the other hand, iris preprocessing aims to detect the circular region surrounding the iris[5]. As a result, we adopted a algorithmbased codifier to encode both fingerprint and iris features, obtaining a unified template. Then we obtain matching score of individual biometric. Successively, the HD on the fused template has been used for the similarity index computation[12].

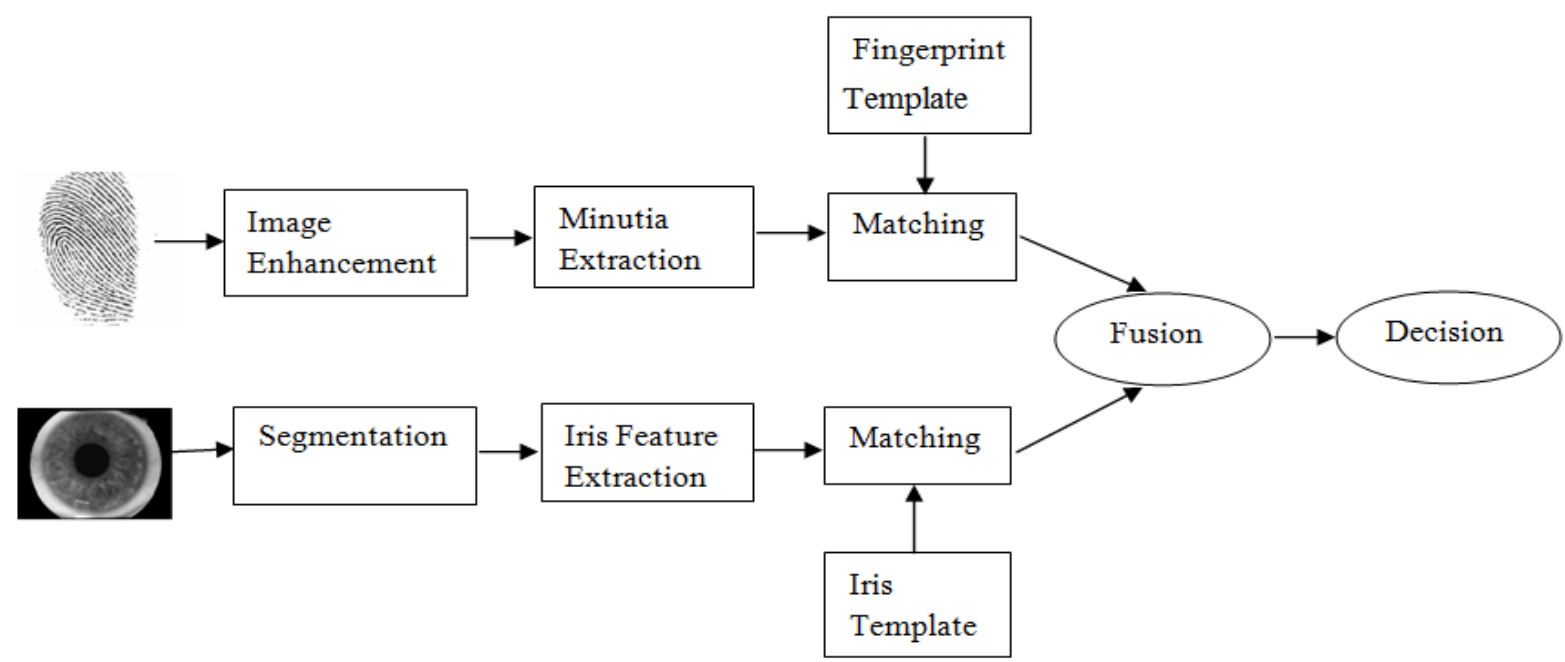

Fig 1. block diagram of multimodal biometric system . 
In this structure of multimodal biometric identification system, two biometric models have been fused.

1. Fingerprint Recognition

2. Iris Recognition

Each individual biometric model has to go under different level of processing like Preprocessing, Normalization, Feature Extraction and Matching to get proper Matching score of both biometric individually and we apply fusion technique at matching score level with obtained matching score. The system can work either sequentially or parallel.

\section{Fingerprint Recognition}

Fingerprint recognition is the most consistent biometric modality in use, The main reason behind the use of fingerprint biometric is that it is the most proven technique to identify the individual. Digital fingerprints are more convenient and less disturbing than most of the other biometric methods and they are already accepted as an immutably single identifier. The fingerprint is basically the combination of ridges and valleys on the surface of the finger. However, revealed by rigorous research on fingerprint recognition, fingerprints are not distinguished by their ridges and furrows, but by Minutia, which are typical points on the ridges[11] . There are several minutia types: Termination (the immediate ending of a ridge), Bifurcation (the point on the ridge from which two branches derive),Island (a ridge that commences, travels a short distance and then ends), Ridge enclosures (a single ridge that bifurcates and reunites shortly afterward to continue as a single ridge), Spur (a bifurcation with a short ridge branching off a longer ridge), Crossover or bridge (a short ridge that runs between two parallel ridges)[11][7]. In this paper only two types of minutia points are take into consideration, which are termination and bifurcation. The major steps involved in fingerprint recognition using minutiae matching approach after image acquisition are:

1. Image Enhancement

2. Minutiae Extraction

3. Matching

\subsection{Image Enhancement}

A fingerprint image is corrupted due to various kinds of noises such as creases, smudges and holes. It is almost impossible to recover the true minutia points from the unrecoverable regions The fingerprint image is enhanced to make it clearer for easy further operations by increasing the contrast between ridges and furrows[15]. Image enhancement algorithm is used to improve the clarity of ridges/valley structures of fingerprint images in recoverable regions and to mask out the unrecoverable regions[11]. For this, we adopted an image normalization step using the arithmetic average $\mathrm{M}$ of the image gray levels and its variance $\mathrm{V}$ [12].

$$
\begin{gathered}
M(I)=\frac{1}{H * L} \sum_{i=1}^{H} \sum_{j=1}^{L} I(i, j) \\
V(I)=\frac{1}{H * L} \sum_{i=1}^{H} \sum_{j=1}^{L}(I(i, j)-M)^{2}
\end{gathered}
$$

\subsection{Minutia Extraction}

The endings and bifurcations of the fingerprint images are known as the minutia. The most commonly employed method of minutiae extraction is the Crossing Number $(\mathrm{CN})$ concept.

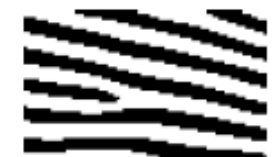

(a) Ridge ending

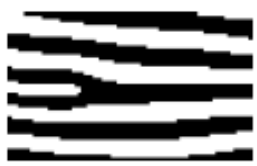

(b) Bifurcation

Fig 2. example of a ridge ending and a bifurcation.

The minutiae are extracted by processing the whole image pixel by pixel using a $3 \times 3$ matrix with the centre element being the focus of each process[14]. The $\mathrm{CN}$ value is then computed, which is defined as half the sum of the differences between pairs of adjacent pixels in the eight-neighborhood[11]. Using the properties of the $\mathrm{CN}$ as shown in table, the ridge pixel can then be classified as a ridge ending, bifurcation or non-minutiae point[7]. For example, a ridge pixel with a $\mathrm{CN}$ of one corresponds to a ridge ending, and a $\mathrm{CN}$ of three corresponds to a bifurcation. The Crossing Number $(\mathrm{CN})$ method is used to perform minutiae extraction[11]. This method extracts the ridge endings and bifurcations from the skeleton image by examining the local neighborhood of each ridge pixel using a $3 * 3$ window[11]. The $\mathrm{CN}$ for a ridge pixel $P$ is given By, 


$$
C N=0.5 \sum_{t=1}^{8}\left|P_{i}-P_{i+1}\right|, \quad P_{9=P_{1}}
$$

where $P i$ is the pixel value in the neighborhood of $P$. For a pixel $P$, its eight neighboring pixels are scanned in an anti-clockwise direction as follows:

\begin{tabular}{|l|c|c|}
\hline$P_{4}$ & $P_{3}$ & $P_{2}$ \\
\hline$P_{5}$ & $P$ & $P_{1}$ \\
\hline$P_{6}$ & $P_{7}$ & $P_{8}$ \\
\hline
\end{tabular}

After the $\mathrm{CN}$ for a ridge pixel has been computed, the pixel can then be classified according to the property of its $\mathrm{CN}$ value. A ridge pixel with a $\mathrm{CN}$ of one corresponds to a ridge ending, and a $\mathrm{CN}$ of three corresponds to a bifurcation[11]. For each extracted minutiae point, the following information is recorded:

- $x$ and $y$ coordinates,

- Orientation of the associated ridge segment, and

- Type of minutiae (ridge ending or bifurcation).
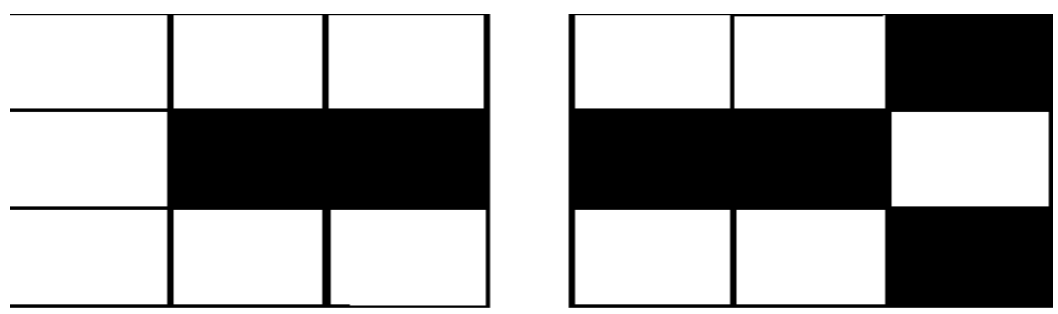

Fig 3. a) 1 Corresponds Ridge ending b) 3 Corresponds Bifurcation

However, there are a few cases where the extracted minutiae do not correspond to true minutiae points in the original image. In addition, it should be noted that in some cases the bifurcation and ridge ending points can be difficult to distinguish between each other[1]. This method not only increases the processing speed, but also reduces the possible error due to scars or noises in the fingerprint image[3]. Minutia extraction is processed in 4 steps:

- Fingerprint ridge thinning

- Minutia marking

- False minutia removal

- Unification of terminations and bifurcations.

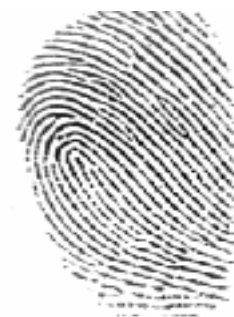

(a)

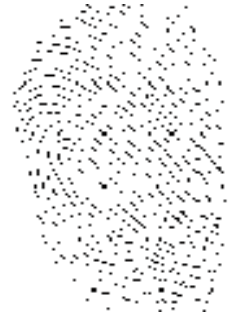

(c)

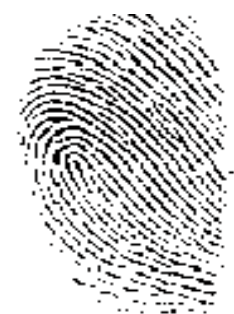

(b)

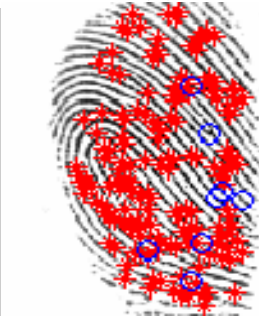

(d)

Fig 4. a)Input image, b) Binariesd image c)Thinned image, d) Minutia points of input image. 


\subsection{Matching}

The minutia match algorithm settles on whether two set of minutia of two fingerprint images belong to the same finger or not .The first step consists in aligning the couple of fingerprint Images to be matched[3]. Thus we choose a minutia from each image and calculate the similarity of the associated ridges[10]. In the matching step, we count the matched minutia pairs of two set of transformed minutia points and we assume identical, two minutia having nearly the same position and direction[3].

\section{IRIS RECOGNITION}

Iris is plainly visible, colored ring that surrounds the pupil which is unique to each individual and remains constant over the life of a person. The iris is the annular ring between the sclera and pupil boundary and contains the flowery pattern unique to each individual. The eyeball has a circular black disk in the center known as pupil. The pupil dilates when exposed to light and contracts in dark. Thus the size of pupil varies with respect to light. There is no detailed correlation between the iris patterns of even identical twins, or the right and left eye of an individual[14]. For our developed approach of iris recognition, the input is an eye image, and the output is the matching score of the iris template. This texture information unique to each individual is extracted from rest of the eye image and is transformed into strip to apply pattern matching algorithm between the database and query images of iris[8]. It compares the difference between a pair of iris representations by computing their Hamming distance using XOR operator[12]. The algorithm consists in 4 steps :

1. Segmentation

2. Normalization

3. Feature Extraction

4. Matching

\subsection{Segmentation}

Iris image must be preprocessed before using it for the recognition purpose since the unwanted data including eyelid, pupil, sclera and eyelashes in the image should be excluded. The preprocessing module for iris was required to perform iris localization, iris normalization and image enhancement[6]. The Iris region is located within the two circles: the iris/sclera boundary and the iris/pupil one. We have used images provided by CASIA (Institute of Automation, Chinese Academy of Sciences)[6]. The images were taken exclusively for the purpose of iris recognition software research and implementation. Infra-red light was used for illuminating the eye, and hence they do not involve any specula reflections. For this reason, we do not proceed for a reflection error's removal. The segmentation step consists in applying Canny edge detection to generate an edge map, to isolate the actual iris region from a captured iris image[5]. In this process, roughly determine the inner and outer boundaries of iris region from iris image, and then accurately locate the fine boundaries in the iris image with raw resolutions[6][10]. Image binarization were next used to extract the eyelid edge information. After this process, histogram equalization was used to implement image enhancement. then using circular Hough transform to detect the iris and pupil boundaries and deduce their radius and centre coordinates[13]. As a result parameters are stored: the radius, and $(\mathrm{x}, \mathrm{y})$ centre coordinates for both circles[12].

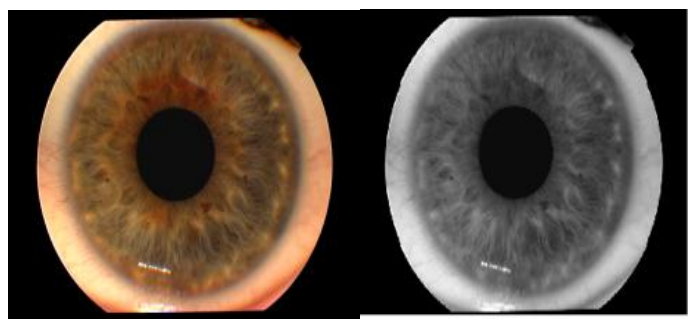

(a)

(b)

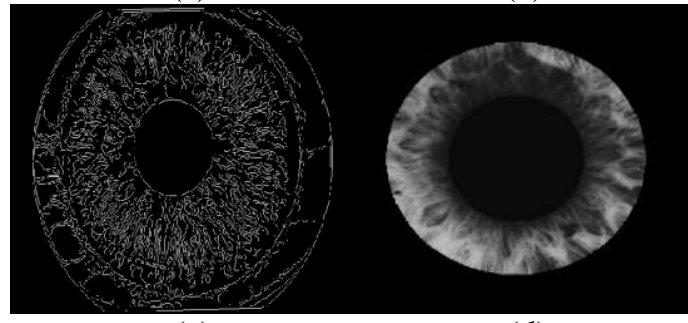

(c)

(d)

Fig 5. a) Original image, b) Gray image, (c) Binerised image, d) Segmented iris imge 


\subsection{Normalization}

Once the iris region is successfully segmented from an eye image, the next stage is to transform the iris region so that it has fixed dimensions in order to allow comparison[6]s. The normalization process will produce iris regions, which have the same constant dimensions. The pupil region is not always concentric within the iris region, For normalization of iris regions a technique based on Daugman's rubber sheet model was employed[10][12]. The centre of the pupil was considered as the reference point, and radial vectors pass through the iris region.

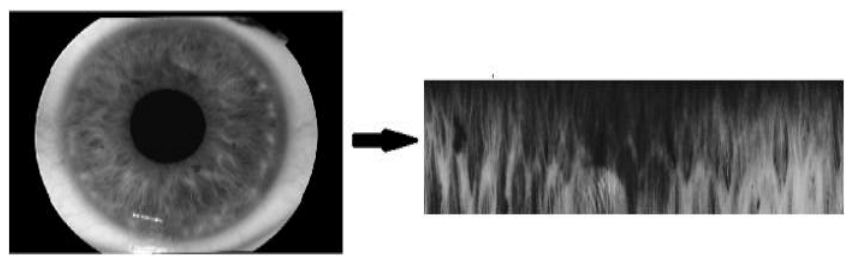

Fig 6. Normalized Iris Image

\subsection{Feature extraction}

The output of the normalization is noise free and is further proceed for feature extraction. In feature extraction, the template which is produced by normalization is converted into binary format for the matching process[1][6]. Feature Extraction is done by using bit plane slicing method. In order to provide accurate recognition of individuals, the most discriminating information present in an iris pattern must be extracted[13]. Only the significant features of the iris must be encoded so that comparisons between templates can be made. Most iris recognition systems make use of a band pass decomposition of the iris image to create a biometric template.

\subsection{Matching}

The comparison is done between iris codes (IC) generated for database and query images using hamming distance approach. In this approach the difference between the bits of two codes are counted and the number is divided by the total number of comparisons[12].

$$
M S_{\text {Iris }}=\frac{1}{N} \sum_{i=1}^{N} A_{i} \oplus B_{i}
$$

where $\mathrm{A}$ is the binary vector (iris code) for database image and $\mathrm{B}$ is the binary vector for query image while $\mathrm{N}$ is the number of elements. This matching score $\left(\mathrm{MS}_{\text {Iris }}\right)$ is used as input for the fusion module where the final matching score is generated.

\section{FUSION OF IRIS \& FINGERPRINT}

No individual trait can provide $100 \%$ accuracy. Further, the results generated from the individual traits are good but the problem arises when the user is not able to give his iris image due to problem in exposure to light. As eye is the most sensitive organ of human body, the problem becomes severe when there exist some eye diseases. Thus in such a situation an individual cannot be recognized using the iris patterns and the biometric system comes to a standstill. Similarly, the problem faced by fingerprint recognition system is the presence of scars and cuts. The scars add noises to the fingerprint image which cannot be enhanced fully using enhancement module. Thus, the system takes noisy fingerprint as input which is not able to extract the minutiae points correctly and in turn, leads to false recognition of an individual. Thus to overcome the problems faced by individual traits of iris and fingerprint, a novel combination is proposed for the recognition system[5][10]. The integrated system also provide anti spoofing measures by making it difficult for an intruder to spoof multiple biometric traits simultaneously. Scores generated from individual traits are combined at matching score level using weighted sum of score technique[17]. Sum rule is used for the fusion of iris \& fingerprint Matching score. A fusion system consist of two modules fusion and decision module. In the fusion module is well understood that need to normalize scores before combining them, that is seek to transform individual scores from each subsystem before combining[17]. In fact score from each subsystem can be of different nature. To solve this problem, the normalization transforms the score into common range include between 0 and 1 [17][13]. After that we use score combination method using a simple sum. Combining the average scores is to compute as,

$$
s=\frac{1}{N} \sum_{i=1}^{N} s_{i}
$$




\section{CONCLUSION}

In this paper we introduced an innovative multimodal biometric identification system based on iris and fingerprint traits. Iris is the unique organ and remains stable throughout the lifetime. Fingerprint is more selective as compare to other biometric. That's why we take these traits. The features are extracted from iris \& fingerprint image. Then template is formed \& compare with stored one with the help of hamming distance. The Multimodal identification system is fused at Matching level fusion using sum rule at the verification stage. The results show improvements in the fingerprint verification phase and iris segmentation process. The proposed system works fine as it's FAR \& FRR. The accuracy of the multimodal system is very high. So that multimodal biometric system has the potential to overcome the limitations of any individual biometric system. The multimodal biometric system provides more security to electronic data and resource access from unauthorized user. The performance of the multimodal biometric identification system shows significant improvement.

\section{Acknowledgements}

I am sincerely thankful to my guide Prof. Dr. A. S. Abhyankar for his relevant help, encouragement and providing the necessary guidance. I am also proud to thank our HOD (E\&TC Department) Prof. P. D. Khandekar and our Principal Prof. Dr. B. S. Karkare for moral support. I am really thankful to all professors of VIIT for their guidance. Without their help it was tough job for me.

\section{Journal Papers:}

\section{REFERENCES}

[1] L. Hong, A. Jain, "Multimodal Biometrics", chapter 16 in A.Jain, R. Bolle , S. Pankanti, "Biometrics: Personal Identification in Networked Society", Kluwer Academic Publishers, Norwell, 1999.•

[2] S. Prabhakar, S. Pankanti, and A. K. Jain, "An Introduction to Biometric Recognition," IEEE Transactions on circuits and systems for video technology, VOL. 14, NO. 1, January 2004.

[3] Vincenzo Conti, Carmelo Militello, Filippo Sorbello, and Salvatore Vitabile, "A Frequency-based Approach for Features Fusion in Fingerprint and Iris Multimodal Biometric Identification Systems," IEEE transactions on systems, man, and cybernetics-part c: applications and reviews, VOL. 40, NO. 4, july 2010.

[4] Anil Jain Sharath Pankanti,Dept. of Computer Science \& Engg. Exploratory Computer Vision Grp, "Fingerprint Classification and Matching,"

[5] R. Wildes, "Iris Recognition: An emerging biometric technology" Proceedings of the IEEE, vol.85, no.9, September 1997.

[6] Arun Ross, Anil Jain, Jain-Zhong Qian - Information Fusion in Biometricsll Appeared in Proc. of 3rd Int'l Conference on Audioand Video-Based Person Authentication (AVBPA), pp. 354-359, Sweden, June 6-8, 2001.

[7] A. K. Jain, A. Ross, and S. Pankanti, "An introduction to biometric recognition," IEEE Trans. Circuits Syst. Video Technol., vol. 14, no. 1, pp. 4-20, Jan. 2004.

[8] Chan K.C, Y.S Moon and Cheng, "Fast fingerprint verification using subregions of fingerprint images", IEEE Trans.on Circuits and Systems for video technology, vol 14, Jan 2004.

[9] F. Besbes, H. Trichili, and B. Solaiman, "Multimodal biometric system based on fingerprint identification and Iris recognition," in Proc. 3rd Int. IEEE Conf. Inf. Commun. Technol.: From Theory to Applications (ICTTA 2008), pp. 1-5. DOI: 10.1109/ICTTA.2008.4530129.

[10] L. Ma, Y. Wang and D. Zhang, -Efficient iris recognition by characterizing key local variations\| IEEE Trans. Image Process., vol. 13, no. 6, pp. 739-750, Jun. 2004.

[11] Y. Zhu, T. Tan, and Y. Wang, "Biometric personal identification on iris patterns," in Proc. 15th Int. Conf. Pattern Recogn., 2000, vol. 2, pp. 805-808.

[12] L. Hong, Y.Wan, and A. Jain, "Fingerprint image enhancement, algorithm and performance evaluation," IEEE Trans. Pattern Anal. Mach. Intell., vol. 20, no. 8, pp. 777-789, Aug. 1998.

[13] S. Prabhakar, A. K. Jain, and J.Wang, "Minutiae verification and classification,"presented at the Dept. Comput. Eng. Sci., Univ. Michigan State,East Lansing, MI, 1998.

[14] N. K. Ratha, R. M. Bolle, V. D. Pandit, and V. Vaish, "Robust fingerprint authentication using local structural similarity," in Proc. 5th IEEE Workshop Appl. Comput. Vis., Dec. 4-6, 2000, pp. 29-34. DOI 10.1109/WACV.2000.895399.

[15] Fingerprint Verification Competition FVC2002. (2009, Nov.). [Online]. Available: http://bias.csr.unibo.it/fvc2002/

[16] J. Cui, J. P. Li, and X. J. Lu, "Study on multi-biometric feature fusion and recognition model," in Proc. Int. IEEE Conf. Apperceiving Comput. Intell. Anal. (ICACIA), 2008, pp. 66-69. DOI: 10.1109/ICACIA.2008.4769972.

[17] V. Conti, G. Milici, P. Ribino, S. Vitabile, and F. Sorbello, "Fuzzy fusion in multimodal biometric systems," in Proc. 11th LNAI Int. Conf. Knowl.Based Intell. Inf. Eng. Syst. (KES 2007/WIRN 2007), Part I LNAI 4692. B. Apolloni et al., Eds. Berlin, Germany: Springer-Verlag, 2010, pp. 108-115.

[18] G. Aguilar, G. Sanchez, K. Toscano, M. Nakano, and H. Perez, "Multimodal biometric system using fingerprint," in Proc. Int. Conf. Intell. Adv.Syst. 2007, pp. 145-150. DOI:10.1109/ICIAS.2007.4658364.

[19] V. C. Subbarayudu and M. V. N. K. Prasad, "Multimodal biometric system," in Proc. 1st Int. IEEE Conf. Emerging Trends Eng. Technol., 2008, pp. 635-640. DOI 10.1109/ICETET.2008.93.

[20] F. Yang and B. Ma, "A new mixed-mode biometrics information fusion based-on fingerprint, hand-geometry and palm-print," in Proc. 4th Int. IEEE Conf. Image Graph., 2007, pp. 689-693. DOI:10.1109/ICIG.2007.39.

[21] BATH Iris Database, University of Bath Iris Image Database. (2009, Nov.). [Online]. Available: http://www.bath.ac.uk/eleceng/ research/sipg/irisweb/

\section{Books:}

[22] A.Ross, K. Nandakumar, A. Jain, "Handbook of Multibiometrics", Springer, New York, 2006•

[23] "Multimodal Biometric Systems", chapter7 in D. Maltoni , D. Maio , A.K. Jain, S . Prabhakar, "Handbook of Fingerprint Recognition”, Springer-Verlag ,New York,2003.•“ 


\section{Theses:}

[24] John Daugman. "How Iris Recognition Work". IEEE Transaction on Circuits and Systems for VIDEO TECHNOLOGY, VOL. 14, No. 1, January 2004.

\section{AUTHOR'S PROFILE}

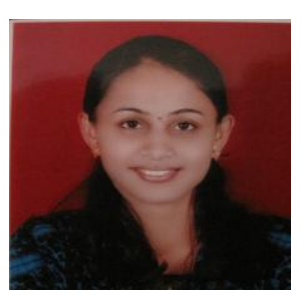

\section{Ms. Priyanka S. Patil}

Received the degree of B.E in Electronics and Telecommunication Engineering from BVCOEW Engineering College, Pune, Maharashtra in 2009 and pursuing M.E. in Electronics \& Telecommunication Engineering from VIIT College of Engineering, Pune. With having 1 year teaching experience.

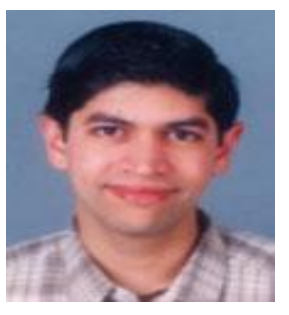

\section{Prof.(Dr.) A. S. Abhyankar}

Ph.D. in (Electrical and Computer Engineering ) Clarkson University, NY, USA, · M.S. in (Electrical and Computer Engineering ) Clarkson University, NY, USA, · B.E. in (E\&TC) University Of Pune. He is Dean Research \& Development Professor, Department of Computer Engineering at VIIT college of Engineering. With teaching \& research experience of 10 years and industrial experience of 2 years. He has published papers in National-International conferences \& International Journals. His Area of interest in Pattern Recognition, Digital Signal and Image Processing, Wavelet Analysis, Biomedical Signals, Biometrics, Bio-informatics. 